\title{
Digital Preservation and Access of Audio Heritage: A Case Study for Phonographic Discs
}

\author{
Sergio Canazza ${ }^{1}$ and Nicola Orio ${ }^{2}$ \\ ${ }^{1}$ Lab. AVIRES, Dept. of Historical and Documentary Science \\ University of Udine, Via Petracco, 8, 33100, Udine (Italy) \\ sergio.canazza@uniud.it \\ ${ }^{2}$ Information Management Systems Research Group, Dept. of Information Engineering \\ University of Padova, Via Gradenigo 6/a, 35100, Padova (Italy) \\ orio@dei.unipd.it
}

\begin{abstract}
We investigate differences among the approaches to the digitization of phonographic discs, using two novel methods developed by the authors: a system for synthesizing audio signals from still images of phonographic discs and a tool for the automatic alignment of audio signals. Results point out that this combined approach can be used as an effective tool for the preservation of and access to the audio documents.
\end{abstract}

Keywords: Audio cultural heritage, Audio documents access, Audio alignment.

\section{Introduction}

The importance of transferring audio analogue documents into the digital domain, known as active preservation, has been recognized by the international archive community, which provided a number of guidelines in particular for carriers in risk of disappearing. Within this group of documents the sound recordings on discs have been the most spread in the period from 1898 until about 1990. This wide time span in which these formats have been developed makes it difficult to select the correct playing format for the carriers, which may differ in speed, number of tracks, and chemistry characteristics. In order to provided the most complete information about the carrier, the auditory information can be augmented with cross-modal cues. We believe that it is interesting to deal with other information regarding the carrier corruption and imperfection occurred during the $\mathrm{A} / \mathrm{D}$ conversion. This work describes different approaches for the A/D transfer of phonographic discs and provides two case studies where the similarities and differences of the approaches to re-recording are highlighted.

\section{Phonographic Discs Re-recording Systems}

Four typologies of playing equipment exist.

Mechanic. It uses a mechanical phonograph, the most common device for playing recorded sound from the 1870s through the 1950s, where the stylus is used to vibrate a diaphragm radiating through a horn. 
Electro-Mechanical. Turntable drive systems with pickup (piezo-electric crystal or magnetic cartridges), operating on the physics principle of electromagnetic induction.

Opto-Mechanical. A laser turntable is a phonograph that plays gramophone records using a laser device as the pickup. Laser pickup reduces many problems associated with physical contact of the stylus. Unfortunately, the it is susceptible to damage and debris and very sensitive to surface reflectivity.

Opto-Digital. Digital image processing techniques can be applied to the problem of extracting audio data from recorded grooves, acquired using an electronic camera or other imaging system. The images can be processed to extract the audio data also in the case of a broken disc. In literature there are several approaches to this problem [1].

The authors have developed an HW/SW system (Photos of GHOSTS: PoG [2]) which: a) is able to recognize different rpm and to perform tracks separation; b) works with both low-cost hardware (starting from a $2 \mathrm{D}$ reconstruction of the grooves) and not-trained personnel; c) is robust with respect to dust and scratches; d) outputs denoised and pitch corrected sound by means of novel restoration algorithm [3]; e) can apply hundreds of different equalization curves. The software automatically finds the disc center and radius from the scanned data, then performs groove rectification and track separation. Starting from the light intensity curve of the pixels in the image, the groove is modeled and the audio samples are obtained.

\section{Case Study}

As case study, we selected the double-sided $78 \mathrm{rpm} 10$ " shellac disc recorded in New York on February the $23^{\text {rd }} 1928$ :

- $\quad$ Rosina Gioiosa Trubia - Sta terra nun fa pi mia (This land is not for me) (R. Gioiosa, arr. R. Romani). Brunswick 58073B (E 26621/2);

- Rosina Gioiosa Trubia - Mi vogghiu maritari (I want to get married) (R. Gioiosa, arr. R. Romani). Brunswick 58073A (E 26617/8).

The audio signal was digitized using a sampling rate of $48 \mathrm{kHz}$ with 24 bit resolution. The re-recording has been carried out in four ways:

a) mechanical: phonograph His Master Voice, Monarch model, equipped with a pickup HMV Exhibition and a horn Columbia. The re-recording was carried out in a 4 $\mathrm{m}^{2}$ room, using a microphone Rhøde NT23, in parallel to the horn axis, $7.5 \mathrm{~cm}$ away and central respect to the horn. By means of a Motu 828 MkII, the signal is transferred in the digital domain.

b) Electro-mechanical: turntable Diapason model 14-A, arm Diapason, pickup Shure M44/7, Stylus Expert. The setting was: $78.26 \mathrm{rpm} ; 3.5 \mathrm{mil} ; 4 \mathrm{~g}$; truncated elliptical; FFRR equalization curve. Prism A/D Converter Dream AD-2.

c) Opto-mechanical: player laser ELP mod. LT-1XRC. The setting was: $78.26 \mathrm{rpm}$, FFRR equalization curve. Prism A/D Converter Dream AD-2.

d) Opto-digital: $P o G$ system, 4800 dpi, 8 bit grayscale, no digital correction. 

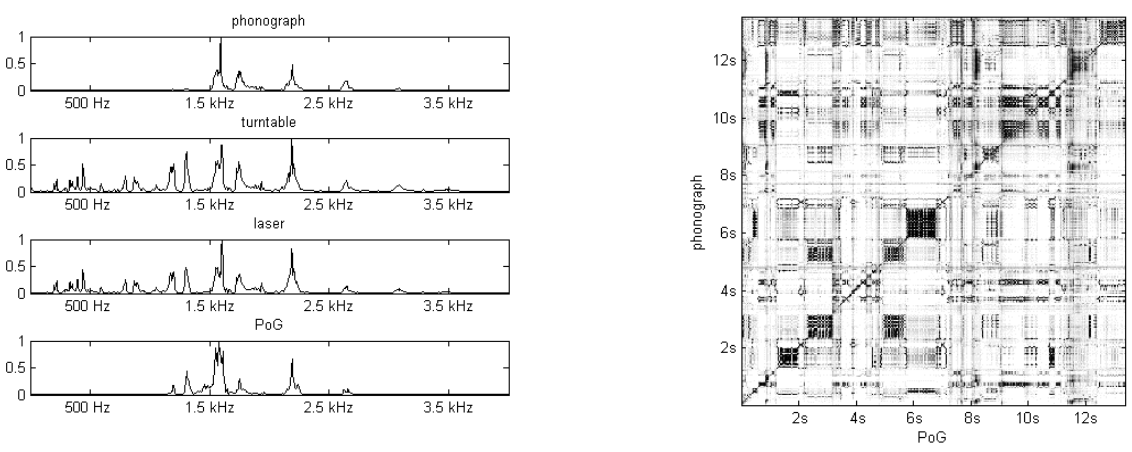

Fig. 1. Left: periodograms of about 14 seconds of Sta terra nun fa pi mia, taken with the four re-recording systems (the amplitudes have been normalized to ease the comparison). Right: Graphical representation of the local similarities between PoG and phonograph recordings.
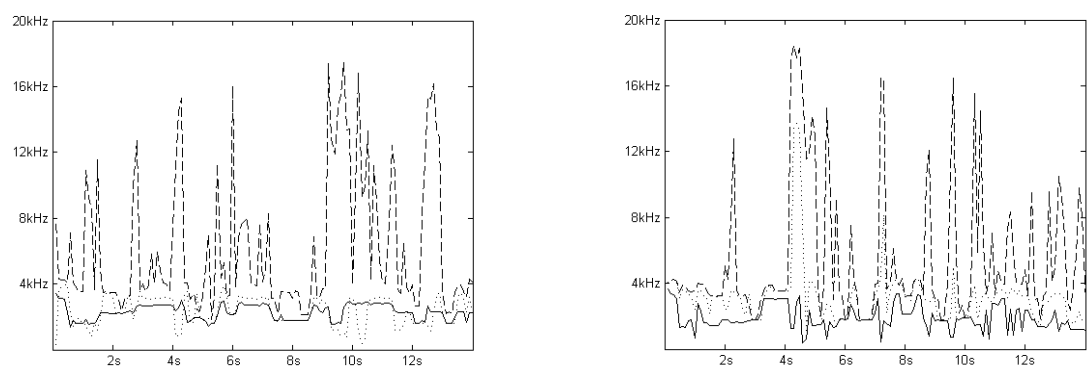

Fig. 2. Rolloff of three aligned signals for Sta terra nun fa pi mia (left) and Mi vogghiu maritari (right): PoG (solid), turntable (dashed), and laser (dotted)

The four audio signal spectral density estimations (periodograms) of the first case study, shown in Fig. 1 (left), highlight that, even if turntable and laser are more sensitive, both to the original signal and to the artifacts (scratch and dust) on the carrier, the four systems give comparable results. A closer analysis of the turntable spectrum, showed a peak at about $11 \mathrm{~Hz}$, which is due to the mechanical vibrations of the arm and probably to a resonance between the arm and the engine. The same peak is not observed in the three other spectra. Similar considerations apply to the second case study.

An audio alignment method [4] was used to compare the similarities between these audio signals. At first we compared the playback speed of the mechanical equipment with $P o G$, which is a useful reference because it is not subject to mechanical variations. For the two case studies, we noticed that all the systems had constant speed, with no variation from the nominal value. Fig. 1 (right) highlights this regular trend for the phonograph, the dark areas on the main diagonal represent a high similarity between the two recordings, while occasional lighter areas represent differences that can be due to a different robustness to carrier corruptions.

Moreover, we focus on the differences between the electro- and opto-mechanical systems, using PoG as a reference, to investigate the possibility to automatically 
recognize the type of modern re-recording system. We computed and aligned the most relevant features proposed in the audio processing literature. In particular, the first four spectral moments (centroid, spread, skewness, and kurtosis), brightness, and spectral rolloff have been computed using an analysis window of 8192 points. As it can be noted from Fig. 2, spectral rolloff is a good indicator of the differences among the recording systems. Moreover, we computed mean and variance of the point to point differences of the spectral rolloffs, using $P o G$ as a reference: (i) turntable versus $P o G$; (ii) laser versus $P o G$. Results are reported in Table. 1. In particular, the value of the variance allows us to identify the kind of re-recording system.

Table 1. Mean and variance of the difference between spectral rolloff using $P o G$ as a reference

\begin{tabular}{llll}
\hline Case study & Re-recording systems & Mean & Variance \\
\hline Sta terra nun fa pi mia & turntable & 4287 & 4139 \\
Sta terra nun fa pi mia & laser & 10.32 & 768.4 \\
Mi vogghiu maritari & turntable & 3701 & 4473 \\
Mi vogghiu maritari & laser & 1320 & 1810 \\
\hline
\end{tabular}

\section{Conclusion}

Audio archivists can take advantage from a variety of equipments for the re-recording of phonographic discs. We presented two case studies using the four different paradigms. We propose a combined approach: by applying the procedure described in this work, that is synthesizing an audio signal using $\operatorname{PoG}$ and aligning it to the existing digital documents, it is possible to identify the kind of A/D process, its eventual defects, and retrieve the original analogue recording.

\section{References}

1. Fedeyev, V., Haber, C.: Reconstruction of mechanically recorded sound by image processing. Journal of the Audio Engineering Society 51(12), 1172-1185 (2003)

2. Canazza, S., Ferrin, G., Snidaro, L.: Photos of GHOSTS: Photos of Grooves and HOles, Supporting Tracks Separation. In: Proceedings of XVII CIM Venezia, October 5-17, 2008, pp. 171-176 (2008)

3. Bari, A., Canazza, S., De Poli, G., Mian, G.A.: Toward a Methodology for the Restoration of Electro-Acoustic Music. Journal of New Music Research 30(4), 365-374 (2001)

4. Orio, N., Snidaro, L., Canazza, S.: Semi-automatic metadata extraction from shellac and vinyl discs. In: Proceedings of AXMEDIS 2008, Firenze, Italy, pp. 38-45 (2008) 